This is a self-archived version of an original article. This version may differ from the original in pagination and typographic details.

Author(s): Chang, Zheng; Zhang, Di; Hämäläinen, Timo; Han, Zhu; Ristaniemi, Tapani

Title: Incentive Mechanism for Resource Allocation in Wireless Virtualized Networks with Multiple Infrastructure Providers

Year: 2020

Version: Accepted version (Final draft)

Copyright: (C) 2018 IEEE

Rights: In Copyright

Rights url: http://rightsstatements.org/page/InC/1.0/?language=en

Please cite the original version:

Chang, Z., Zhang, D., Hämäläinen, T., Han, Z., \& Ristaniemi, T. (2020). Incentive Mechanism for Resource Allocation in Wireless Virtualized Networks with Multiple Infrastructure Providers. IEEE Transactions on Mobile Computing, 19(1), 103-115.

https://doi.org/10.1109/TMC.2018.2889046 


\title{
Incentive Mechanism for Resource Allocation in Wireless Virtualized Networks with Multiple Infrastructure Providers
}

\author{
Zheng Chang, Senior Member, IEEE, Di Zhang, Student Member, IEEE, \\ Timo Hämäläinen, Senior Member, IEEE, Zhu Han, Fellow, IEEE, and \\ Tapani Ristaniemi, Senior Member, IEEE
}

\begin{abstract}
To accommodate the explosively growing demands for mobile traffic service, wireless network virtualization is proposed as the main evolution towards 5G. In this work, a novel contract theoretic incentive mechanism is proposed to study how to manage the resources and provide services to the users in the wireless virtualized networks. We consider that the infrastructure providers (InPs) own the physical networks and the mobile virtual network operator (MVNO) has the service information of the users and needs to lease the physical radio resources for providing services. In particular, we utilize the contract theoretic approach to model the resource trading process between the MVNO and multiple InPs. Two scenarios are considered according to whether the information (such as the radio resource they can provide) of the InPs are globally known. Subsequently, the corresponding optimal contracts regarding the user association and transmit power allocation are derived to maximize the payoff of the MVNOs while maintaining the requirements of the InPs in the trading process. To evaluate the proposed scheme, extensive simulation studies are conducted. It can be observed that the proposed contract theoretic approach can effectively stimulate InPs' participation, improve the payoff of the MVNO and outperform other schemes.
\end{abstract}

Index Terms - wireless virtualized network; contract theory; resource allocation; power allocation; user association

\section{INTRODUCTION}

\subsection{Background and Motivation}

The aim of $5 \mathrm{G}$ is to provide ubiquitous connectivity for any type of device and application that may benefit from being connected. To realize the vision of essentially unlimited access to information and sharing of data anywhere and anytime for anyone and anything, recent emerging mobile platforms, such as Software Defined Network (SDN) [1] and Network Function Virtualization (NFV) [2], bring us novel views on the current cellular wireless networks, which urges us to rethink current network infrastructure. The recent advances also open the way to expand SDN/NFV concepts to Radio Access Networks (RANs), creating the Wireless Virtualized Networks (WVNs) framework in which the execution of RAN functions is moved from dedicated telecom hardware to commoditized IT platforms owned by multiple Infrastructure Providers (InPs). In the context of WVN, infrastructure and radio resources can be abstracted, sliced and shared, and a mobile network operator can rent the radio resources in a virtualized manner [3]. Consequently, the overall expenses of wireless network deployment and operation can be significantly reduced [3].

- Z. Chang, D. Zhang, T. Hämäläinen and T. Ristaniemi are with Faculty of Mathematical Information Technology, University of Jyväskylä, P.O.Box 35, FIN-40014 Jyväskylä Finland. E-mail: \{zheng.chang, zhang.d.zhang, timo.t.hamalainen, tapani.ristaniemi \@jyu.fi

- Z. Han is with Electrical and Computer Engineering Department, University of Houston, Houston, TX. E-mail: zhan2@uh.edu.
The emerging cloud computing and virtualization technologies shed light on implementing the SDN/WVN platform. In the WVN, network infrastructures and functionalities are decoupled to maximize their utilization, where the differentiated services can coexist on the same infrastructure. In addition, because the network is expected to be highly heterogeneous and extremely dense, it is natural to consider whether the network infrastructure can be virtualized and provided to whomever wants it and whenever it is acquired. By such, operating the networks is no longer hardware-limited, or even software-limited, as the hardware and software are owned by different and dedicated network InPs. Correspondingly, the traditional two-layer network (network operator/service provider, and users) becomes a multiple-layer vertical architecture, as shown in Fig. 1, where at least three parties are involved. In this architecture, network service provider (NSP) that focuses on providing services to users, the mobile virtual network operator (MVNO) who virtualizes and manages the resources, and the InP that offers the radio resources, are co-existed.

Although the WVN may lower the standard to become a network operator and bring the user many more choices when choosing services, opportunities come with challenges. Apparently, there is a need to explore the interactions and relations among all the involved parties to properly schedule the limited resources with the services. Indeed, it is necessary to introduce an efficient resource virtualization and allocation scheme in the WVN. Moreover, as the InPs cannot directly negotiate with users, it is also very importance to design suitable incentive mechanisms that can en- 
courage the InPs to provide the radio resources. As the InPs are heterogeneous with different preferences for providing services, in terms of operation cost, energy consumption, available spectrum, etc, it is of significance to design a mechanism in which InPs will be stimulated in accordance with their preferences and willingness. Moreover, there is an information asymmetry between the InPs and the MVNO, e.g. the InPs are aware of their own types or preferences while the MVNO may not have that information, which is quite a practical case in the real network. Thus, it is also essential to take into account information asymmetry when modeling the interaction between the InPs and the MVNO.

To this end, we utilize the contract theoretic approach to model the interaction between the InPs and the MVNO, and provide an efficient resource allocation scheme to serve the users. Contract theory is a powerful framework from microeconomics, which provides a useful set of tools for designing incentive mechanisms. Essentially, a contract is a certain type of reward to the employee from the employer in return for its services. Using contract theoretic approaches, one can analyze the interactions between the employer that can offer proper contracts to the employees whose skills may not be known. In the WVN context, this contractual situation can be used to study the interactions between the MVNO (employer) and the InPs (employees) whose certain information may not be unknown to the MVNO. The contract represents performance-reward bundle rewards provided by the MVNO to the specific InPs that provide the required resources and quality-of-service. The main advantages of adopting contract theory in such a scenario include 1) the ability to incorporate resource control and service provisioning in the WVN, 2) notions such as self-revealing contracts suitable to handle information asymmetry between the MVNO and the InPs, 3) the ability to obtain optimal reward and incentive mechanisms that can induce coordination between the InPs.

\subsection{Contribution}

The aim of this paper is to design a resource allocation mechanism to maximize the utility of the MVNO and meanwhile enhance the satisfaction of the InPs. In the considered system, the MVNO needs to rent the physical network (radio resources) from the $\mathrm{InP}$ and operate the network in a virtualized way to provide services to multiple user equipments (UEs). To the best of our knowledge, this work is an early attempt to investigate the relations between the MVNO and InPs, and corresponding resource allocation problems from contract theory and network economic perspectives. Compared to other recent work, our contributions can be summarized as follows:

- Considering an WVN with multiple InPs and an MVNO, we propose to utilize contract theory to model the interactions between the InPs and the MVNO, to provide qualified services to the UEs.

- With the objective to maximize the payoff of the MVNO, which is related to the service quality and utilized resources, we propose an incentive mechanism to motivate InPs to offer their radio resources to provide services. Meanwhile, the objective of each

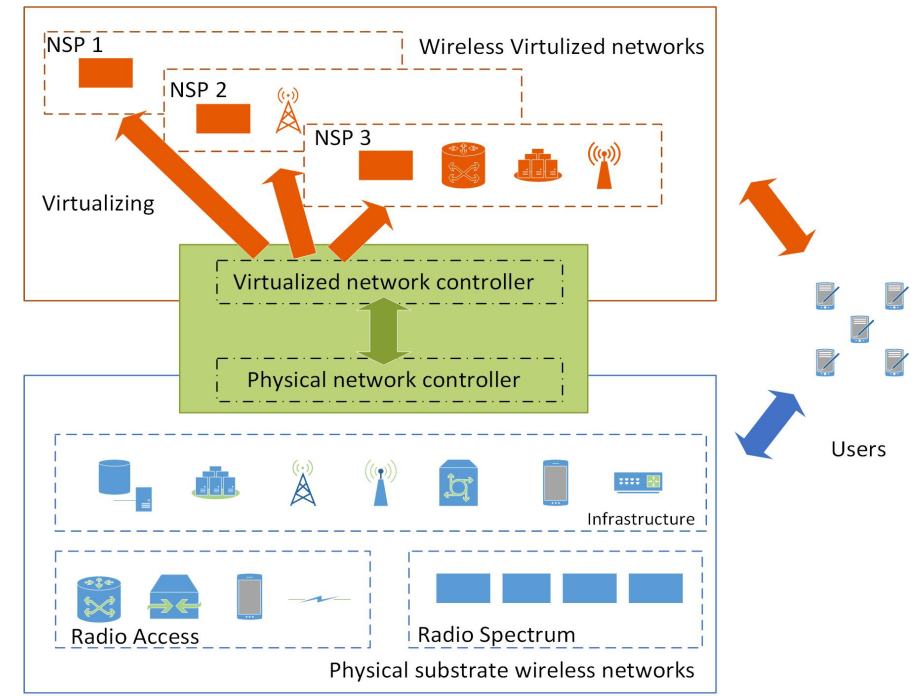

Fig. 1. Wireless Network Virtualization

InP is to maximize its payoff from making the contract with the MVNO and serving the UEs.

- Two information scenarios are considered. We first consider the complete information scenario as a benchmark where the information of the InPs is fully known to the MVNO when designing the contract. Then we consider the contract design in an asymmetric (incomplete) information scenario where the MVNO knows only limited information of the InPs.

- The formulated optimization problem can be considered an user association and power allocation problem in the wireless virtualized network, to optimize the user and InP association and the transmit power in the downlink. After providing its condition of feasibility and optimality, the formulated problem can be addressed to obtain close-to-optimal solutions.

- Numerical results validate the effectiveness of the proposed scheme in the contract design, resource allocation algorithm. It shows that the contract theoretic approach is very attractive for the design of wireless network virtualization for modeling the relations between the MVNO and InP.

\subsection{Related Works}

Recently, there has been growing research interests in enabling network virtualization in wireless networks [4]. In particular, there are considerable efforts dedicated to the resource allocation problems in the WVN. To minimize the operation cost of running the physical network of an InP, in [5], the authors propose a new formulation for the bandwidth allocation and routing problem for multiple WVNs that operate on top of the physical substrate network. A preventive traffic disruption model is also presented for WVNs to minimize the amount of traffic that NSPs have to reduce when substrate link failures happen. In [6], the authors propose a resource allocation scheme for an Orthogonal Frequency Division Multiplexing (OFDM)-based WVN. With the objective of obtaining the energy efficiency in the uplink transmission, a joint power, subcarrier, and 
antenna allocation scheme is proposed to determine the resource allocation in each slice considering the availability of perfect and imperfect channel state information. A downlink resource allocation in a multi-cell WVN is considered in [7] to support the users of different NSPs. In particular, a joint user association, subcarrier, and power allocation algorithm is proposed to maximize the network throughput, while satisfying the minimum required rate of each NSP. The authors of [8] propose a resource allocation scheme for the OFDM-based information-centric WVN. The objective is to maximize the defined payoff of the system considering not only the revenue earned by serving the users, but also the cost of the infrastructure. The authors of [9]-[11] utilized auction theory for resource allocation in WVN. In [12], the authors utilize contract theory to investigate the interaction between NSPs and the MVNO and present a bandwidth provisioning scheme. In [13], virtual resource management for energy optimization is formulated as a three-stage Stackelberg game, and the perfect equilibrium for each stage is analyzed. In [14], the interaction between the MVNOs and users is formulated as a Stackelberg game. The MVNOs can provide data coverage by subleasing the resource from the InPs. The users can choose MVNOs by satisfying their imposed coverage, quality-of-service (QoS) and budget constraints. By apply matching game, the authors of [15] address the formulated service selection and resource purchasing problem in WVN. A Stackelberg game model for interactions between the InPs and MVNOs for resource allocation in downlink OFDM-based wireless virtualization is presented in [16]. The InP acts as a leader while the MVNOs play the roles of the followers. Accordingly, the Stackelberg equilibrium is derived.

It can be well observed that these resource allocation schemes mainly focus on the NSP side. That is, the works are proposed for the MVNO to decide how to slice or allocate resources to each NSP to serve the UEs. Meanwhile, the investigation of resource allocation problems between the MVNO and InPs lacks attention. Contract theory is viewed as an efficient tool for the mathematical modeling of such an interaction [17]. The research on contract theory in wireless communications has been mainly applied to the spectrum trading problems in cognitive radio (CR) [18] [19] and an incentive mechanism to promote Device-to-Device (D2D) communications [20]. In CR networks, the primary users who own the spectrum design the contract and the secondary users who need the spectrum should accept the contract to access the spectrum [18] [19]. In D2D communications, the network operator needs to provide incentives to the D2D transmission to release the traffic load of the cellular Base Station (BS) [20]. Meanwhile, contract theory has also been applied to modeling the service provisioning and resource allocation in cellular networks. In [21] and [22], the authors present the contract theoretic approach for the design of traffic offloading and the caching scheme, respectively. The authors of [23] investigate the user association and spectrum allocation scheme for a heterogeneous network by proposing a contract theory-based scheme. To date, utilizing contract theory to present a thorough analysis of interaction of the InPs and MVNO is full of potential and can complement the current research on WVN, which has not been investigated thus far.

\subsection{Organization}

The remainder of this paper is organized as follows. Section II describes the system model and assumptions. We address the contract formulation and solution with complete information in Section III and the contract formulation and solution with information asymmetry in Section IV. The performance evaluation is presented in Section $\mathrm{V}$, and Section VI concludes the work.

\section{SyStem Model}

\subsection{Wireless Network Virtualization}

WVNs have recently received increasing interest and considered one of the key enablers of the 5G network. In an WVN, physical wireless network infrastructure and radio resources, owned by different InPs, can be abstracted and sliced into virtual wireless network resources, and shared by multiple parties after a certain level of isolation [3]. After resource virtualization, the virtual resources can be offered to end-users via the MVNO or dedicated NSP. In Fig. 1, a simple illustration of an WVN is presented. In this case, we consider the InPs provide physical resources, the MVNO coordinates the virtualized resources, and NSP utilizes the resource to offer service to the users. For service provisioning, the MVNOs in Fig. 1 ask the InPs about the radio resources. Then, the physical radio resources, including spectrum, and infrastructures from different InPs are handled by the physical network controller in the MVNO of which the rule is to lease and virtualize the physical networks into a virtual network based on the requests of NSPs or end-users. The end-users logically connect to the virtual network through which they subscribe to the service, while they physically connect to the cellular network.

In this work, we aim to investigate the relations between the MVNO and InPs for how to incentivize InPs to provide resources for the network and UEs. Therefore, we consider the MVNO can operate the network and connect with the UEs to provide required services. The work can also be extended to the three-level architecture, with the consideration that the NSP consists of a number of the UEs or a group of UEs with same service requirement.

\subsection{System Assumption}

We consider an WVN that includes an MVNO and multiple InPs. The MVNO has $\Gamma$ subscribed UEs, the set of which is denoted as $\mathcal{I}=\{1, \cdots, \Gamma\}$. Each UE $i(i \in \mathcal{I})$ has specific content request $s_{i}$. In order to satisfy UEs' requests, the MVNO will offer a contract that can effectively motivate InPs to offer the specific services. There are totally $J$ InPs, the set of which is denoted as $\mathcal{J}=\{1, \cdots, J\}$. Each InP $j(j \in \mathcal{J})$ owns a set of BSs ${ }^{1}: \mathcal{N}_{j}=\left\{1, \cdots, N_{j}\right\}$ where $n_{j}$ represents BS $n$ of $\operatorname{InP} j$. There are a total of $K=\sum_{j \in \mathcal{J}} N_{j}$ BSs in the considered virtualized wireless network. For the user association, we define a binary variable as follows,

$$
x_{i j}^{n}= \begin{cases}1, & \text { if UE } i \text { assigned with BS } n_{j}, \\ 0, & \text { otherwise. }\end{cases}
$$

1. BSs here represent all the necessary infrastructures, e.g.: radio access network, backhaul, etc. 
TABLE 1

Notations.

\begin{tabular}{|l|l|}
\hline Notation & Description \\
\hline$r_{i}^{k}$ & Channel capacity of UE $i$ \\
\hline$\theta_{i}^{k}$ & Type of BS/InP $k$ w.r.t. UE $i$ \\
\hline$p_{i}^{k}$ & $\begin{array}{l}\text { Transmit power consumption of InP for } \\
\text { serving UE } i\end{array}$ \\
\hline$x_{i}^{k}$ & UE association indicator \\
\hline$U_{k}$ & Payoff of InP $k$ \\
\hline$\varpi_{i}$ & Payoff of MVNO for serving UE $i$ \\
\hline$N_{i}^{k}$ & Number of type- $\theta_{i}^{k}$ InPs \\
\hline$\pi_{i}^{k}$ & Utility of InP $k$ for serving UE $i$ \\
\hline$e_{i}^{k}$ & Cost of InP $k$ for serving UE $i$ \\
\hline$D_{i}$ & Experienced delay of UE $i$ \\
\hline$Q_{i}$ & Service quality of UE $i$ \\
\hline
\end{tabular}

Other key notations are presented in Table 1. As the MVNO dominates the trading process, we model the resource allocation of the WVN as a monopoly market, in which the MVNO acts as the employer, and the InPs act as the employees.

\subsection{Channel Model}

For the MVNO, it is necessary to investigate the characteristics of link between UE $i$ and BS $n_{j}$ in order to offer suitable contract for the InPs and provide services to the UEs. $r_{i j}^{n}, i \in \mathcal{I}, j \in \mathcal{J}, n \in \mathcal{N}_{j}$ is defined as the channel capacity between UE $i$ and BS $n_{j}$ :

$$
r_{i j}^{n}=w_{i j}^{n} \cdot \log _{2}\left(1+p_{i j}^{n} \cdot \frac{\left|h_{i j}^{n}\right|^{2}}{\mathrm{I}_{i j}+\mathrm{I}_{i j^{\prime}}+\sigma^{2}}\right),
$$

where $w_{i j}^{n}$ is the channel bandwidth offered by BS $n_{j}$ for $\mathrm{UE} i, p_{i j}^{n}$ is the transmit power needed by UE $i$ for the specific requested service with data size $s_{i}$ with BS $n_{j}, \sigma^{2}$ is the noise variance of zero-mean additive white Gaussian noise (AWGN), $\left|h_{i j}^{n}\right|^{2}$ is the channel gain between UE $i$ and BS $n_{j} . \mathrm{I}_{i j}$ and $\mathrm{I}_{i j^{\prime}}$ denote the interference come from the BSs inside and outside of $\operatorname{InP} j$, respectively. Universal frequency reuse is considered such that an UE receives only interference from the BSs other than its serving BS. As discussed in [4], because it is hard to coordinate the transmission among different InPs, we consider the InPs are operating on different licensed spectrum in the considered virtualized wireless network. Therefore, $\mathrm{I}_{i j^{\prime}}$ is assumed to be neglected, and $\mathrm{I}_{i j}$ can be given as

$$
\mathrm{I}_{i j}=\sum_{n^{\prime} \in \mathcal{N}_{j} \backslash n} p_{j}^{n^{\prime}}\left|h_{i j}^{n^{\prime}}\right|^{2}, \forall i \in \mathcal{I}, \forall n \in \mathcal{N}_{j},
$$

where $p_{j}^{n^{\prime}}$ denotes the transmit power of BS $n^{\prime}$ of $\operatorname{InP} j$ which can cause interference to UE $i .\left|h_{i j}^{n^{\prime}}\right|^{2}$ is the corresponding channel gain.

In this work, without loss of generality, we consider $w_{i j}^{n}=1$, which indicates that the spectrum bandwidth for an individual UE is fixed and not optimized. Without loss of generality, we assume $\sigma^{2}=1$ is also identical for all InPs. We assume the channel is a quasi-static Rayleigh fading channel. As the channel state information (CSI) is known to the $\mathrm{BSs}$ owned by the InPs, it is considered the private information of each InP and is not directly available globally or to the MVNOs. In addition, $p_{i j}^{n}$, which is the transmit power needed by UE $i$ from BS $n$ of $\operatorname{InP} j$, is known to InP $j$ but is not accessible to the rest of the network and the MVNO. Thus, the transmit power is also regarded as the private information of the InPs. This information can be measured locally but is not available on the global network. According to the difference in channel quality, we classify the BSs into different categories (types- $\theta$ ):

$$
\theta_{i j}^{n} \triangleq \frac{\left|h_{i j}^{n}\right|^{2}}{\sigma^{2}}
$$

which captures all the private information of $\mathrm{BS} n_{j}$ related to its transmission link with UE $i$. It can be found that a larger $\theta_{i j}^{n}$ indicates a better channel condition. Moreover, the delay that UE $i$ may experience can be expressed as

$$
D_{i}=\frac{s_{i}}{\sum_{n \in \mathcal{N}_{j}} \sum_{j \in \mathcal{J}} x_{i j}^{n} \cdot r_{i j}^{n}}, \forall i \in \mathcal{I} .
$$

We denote $Q_{i}$ as service quality for UE $i$, which is inversely proportional to the service delay:

$$
Q_{i}=\frac{\alpha}{D_{i}}, \alpha>0, \forall i \in \mathcal{I},
$$

where $\alpha$ is the reverse function parameter, and $\alpha>0$. We can clearly see that less service delay (lower $D_{i}$ value) leads to higher service quality (larger $Q_{i}$ value).

\subsection{Type and Payoff Model of InPs}

According to the definition in (4), $\theta_{i j}^{n}$ information can be measured only locally by $\operatorname{InP} j$. By classifying the InP type according to its BS, we can have the following definition of InP type.

Definition 1. For specific UE $i$, there are a total of $K=$ $\sum_{j \in \mathcal{J}} N_{j}$ BSs available for connecting, where $k \in \mathcal{K}=$ $\{1, \cdots, K\}$. The channel quality between $B S n_{j}$ and UE $i$ are sorted in an ascending order and classified into $K$ types: type$1, \cdots$, type $-k, \cdots$, type- $K . \theta_{i}^{k}$ is denoted as the type of BS for specific UE $i$ and follows

$$
\theta_{i}^{1}<\cdots<\theta_{i}^{k}<\cdots \theta_{i}^{K}, \forall i \in \mathcal{I} .
$$

Specifically, we refer to InP $j$ as a type- $\theta_{i}^{k} \operatorname{InP}$, if for $B S n_{j} \in \mathcal{N}_{j}$, the channel between BS $n_{j}$ and UE $i$ is $\theta_{i}^{k}=\theta_{i j}^{n}$. A larger $\theta_{i}^{k}$ implies more willingness to contribute to the services. We define $\Theta_{i}=\left\{\theta_{i}^{1}, \cdots, \theta_{i}^{k} \cdots \theta_{i}^{K}\right\}$ as the set of sorted BSs for UE $i$.

For each UE, multiple types of BSs per InP coexist. We can use Definition 1 to transfer the types of BSs with respect to the UEs to the types of InP. As there are multiple BSs within one InP, from the UE point-of-view, one InP can be considered to have different types. Then, it is reasonable for the MVNO to offer different contracts to one InP according to its service capabilities (type) for different UEs. For example, when InP 1 has two types of BSs $\theta_{i}^{1}$ and $\theta_{i}^{2}$ for UE $i, \operatorname{InP} 1$ can be classified into type- $\theta_{i}^{1}$ and type$\theta_{i}^{2}$. The MVNO offers two contracts to InP 1 accordingly. According to this definition, we can use BS $k$ instead of BS 
$n_{j}$. Correspondingly, we can use $p_{i}^{k}\left(p_{i j}^{n}\right), r_{i}^{k}\left(r_{i j}^{n}\right)$, and $x_{i}^{k}$ $\left(x_{i j}^{n}\right)$ instead.

In this work, the objective of each InP is to maximize its payoff from making a contract with the MVNO and serving the UEs. The payoff of the InP consists of two parts. One is the achieved revenue from selling to MVNO and the cost for providing services to the UEs. First, we can define the cost for serving UE $i$. Such a cost consists of a fixed maintenance cost for BS operation $e_{o}$ and a quality-related cost $\Psi\left(p_{i}^{k}\right)$, i.e.,

$$
e\left(x_{i}^{k}, p_{i}^{k}\right)=x_{i}^{k}\left(e_{o}+\Psi\left(p_{i}^{k}\right)\right),
$$

where $e_{o} \geq 0$ is the fixed cost (e.g., the maintenance cost of the BS) and $\Psi\left(p_{i}^{k}\right)$ is related to the power consumption, e.g., a linear function w.r.t the power consumption.

Then, we define the utility/revenue of an InP by selling the resources to the MVNO to serve UE $i$ as follows,

$$
\pi\left(x_{i}^{k}, \theta_{i}^{k}, p_{i}^{k}\right)=\theta_{i}^{k} x_{i}^{k} \gamma R\left(p_{i}^{k}\right),
$$

where $\gamma$ is the unit cost, and $R\left(p_{i}^{k}\right)$ is the payment of the MVNO in the contract with the InPs. We consider the payment is related to the resource (transmit power) usage of the InP. For the fairness consideration, as well as economic reasons ( e.g., the MVNO may want to serve more UEs), for each UE, the dedicated transmit power may not be too large when the QoS can be guaranteed. Therefore, the function $R(x)$ is not desired to be a linear increasing function. A logarithm function $\operatorname{cog}_{2}(1+x), \forall x \geq 0$ where $c$ is a constant, is used here to characterize the payment of the MVNO, which is an increasing, strictly concave function. Note that (9) is an example for defining the utility and it can be defined in some other ways as well. The payoff of InP $k$, which is the difference between the selling price and the cost, can be modeled as,

$$
\begin{aligned}
U_{k} & =\sum_{i \in \mathcal{I}}\left(\pi\left(x_{i}^{k}, \theta_{i}^{k}, p_{i}^{k}\right)-e\left(x_{i}^{k}, p_{i}^{k}\right)\right) \\
& =\sum_{i \in \mathcal{I}} x_{i}^{k}\left(\theta_{i}^{k} \gamma R\left(p_{i}^{k}\right)-e_{o}-\Psi\left(p_{i}^{k}\right)\right) .
\end{aligned}
$$

Given the payoff function in (10), the InP chooses the contract bundle that maximizes InP's own payoff.

\subsection{Payoff Model of the MVNO}

The payoff of the MVNO is defined as the difference between its utility obtained from its served UEs and the cost paid to the InPs. First, we define utility/revenue $\Phi_{i}$ brought by serving UE $i$ as a linear monotonically increasing function of service quality $Q_{i}$. When the service quality requirement is increased, the UE needs to pay more to the MVNO. $\beta$ is defined as the linear parameter for evaluating the relationship between revenue and service quality. So the utility of the MVNO obtained by serving UE $i$ is defined as:

$$
\Phi(\boldsymbol{x}, \boldsymbol{p})=\beta \cdot Q_{i}=\alpha \beta\left(\frac{\sum_{j \in \mathcal{J}} \sum_{k \in \mathcal{N}_{j}} x_{i}^{k} \cdot r_{i}^{k}}{s_{i}}\right), \forall i \in \mathcal{I},
$$

where $\mathbf{x}$ is the set of $x_{i}^{k}$, and $\mathbf{p}$ is the set of $p_{i}^{k}$. Correspondingly, the cost of the MVNO can be considered the utility of the InPs. Therefore, from the MVNO's perspective, we define the payoff brought by serving UE $i$, denoted by $\varpi_{i}$, as the difference between the revenue and cost, i.e.,

$$
\begin{aligned}
\varpi_{i} & =\frac{\sum_{j \in \mathcal{J}} \sum_{k \in \mathcal{N}_{j}} \alpha \beta x_{i}^{k} \cdot r_{i}^{k}}{s_{i}}-\sum_{j \in \mathcal{J}} \sum_{k \in \mathcal{N}_{j}} \pi\left(x_{i}^{k}, \theta_{i}^{k}, p_{i}^{k}\right) \\
& =\sum_{j \in \mathcal{J}} \sum_{k \in \mathcal{N}_{j}} x_{i}^{k}\left(\frac{\alpha \beta r_{i}^{k}}{s_{i}}-\theta_{i}^{k} \gamma R\left(p_{i}^{k}\right)\right), \forall i \in \mathcal{I} .
\end{aligned}
$$

To simplify the analysis, we consider $\alpha=1, \beta=1, s_{i}=1$, and $\gamma=1$. We can further simplify (12) as

$$
\varpi_{i}=\sum_{k \in \mathcal{N}_{j}} \sum_{j \in \mathcal{J}} x_{i}^{k}\left(r_{i}^{k}-\theta_{i}^{k} R\left(p_{i}^{k}\right)\right) .
$$

Instead of offering the same contract to all InPs, the MVNO provides different contract bundles to different InPs according to InP's type $\theta_{i}^{k}$. The InPs are free to accept or decline any type of contract based on their own evaluation.

\subsection{Contract Formulation}

After introducing the payoff of the MVNO and InPs in (10) and (12), we are ready to present the contract-based incentive mechanism for resource allocation in the considered virtualized networks. In general, contract theory studies how the economic decision-makers construct their contractual arrangements, in the presence of full or asymmetric (private) information [17]. In our case, the types of wireless links between the UEs and the BSs are considered private information that can be measured locally and not directly available on the global level. The MVNO may not know the type of each particular channel and needs to design a contract that will be suitable for all the InPs. Because the MVNO dominates the trading process, we model the resource allocation process as a monopoly market, in which the MVNO acts as the employer and the InPs act as the employees. The employer determines the contract, which specifies the relationship between the employees' performance and the rewards. The contract should identify the specific UE$\mathrm{BS} / \mathrm{InP}$ association, for each possible transmit power level. The MVNO assigns the contract items to maximize its payoff in (12) and pass these items to the InPs. Once the contract is specified, each InP selects the contract item that maximizes the InP's payoffs in (10).

To obtain a successful service for specific UE $i$, the contract offered by the MVNO will be highly based on the quality $Q_{i}$, which is related to $p_{i}^{k}$, type $\theta_{i}^{k} \in \Theta_{i}$, and $x_{i}^{k}$. Recalling (5) and (6), we can rewrite the service quality as

$$
Q(x(\boldsymbol{\theta}), p(\boldsymbol{\theta}))=\sum_{k \in \mathcal{K}} x_{i}^{k} r_{i}^{k}, \forall i \in \mathcal{I} .
$$

As we can see from (10), (13), and (14), the payoff and service quality of the system depend on two factors: $x_{i}^{k}$ and $p_{i}^{k}$. Therefore, we write the contract designed for the type $-\theta_{i}^{k}$ InP

$$
\begin{aligned}
\mathbb{C} & =\left\{\left(x\left(\theta_{i}^{k}\right), p\left(\theta_{i}^{k}\right)\right), \forall \theta_{i}^{k} \in \Theta_{i}, \forall i \in \mathcal{I}\right\} \\
& =\left\{\left(x_{i}^{k}, p_{i}^{k}\right), \forall k \in \mathcal{K}, \forall i \in \mathcal{I}\right\} .
\end{aligned}
$$

An InP of type- $\theta_{i}^{k}$ selects the contract item $\left(x_{i}^{k}, p_{i}^{k}\right)$ by maximizing its own payoff, i.e., 


$$
\left(\tilde{x_{i}^{k}}, \tilde{p_{i}^{k}}\right)=\arg \max _{\left(x_{i}^{k}, p_{i}^{k}\right)} \pi\left(x_{i}^{k}, \theta_{i}^{k}, p_{i}^{k}\right)-e\left(x_{i}^{k}, p_{i}^{k}\right) .
$$

(16) shows that the contract items include the user association as well as the transmit power level. The proposed contract of the MVNO needs to ensure win-win trading between the MVNO and the InPs, and the InPs have the incentive to provide services to the UEs.

Next, the goal is to propose an optimal contract design. A contract is considered as optimal if it yields the maximum payoff for the MVNO. Different information scenarios may result in different optimal contracts. Specifically, we will consider two information scenarios based on whether the channel information is globally available or not.

- In a scenario with complete information, the types of all InPs are known to the MVNO, which is possible to be realized by the feedbacks of the UEs.

- In a scenario with asymmetric information, the types of InPs are not known to the MVNO. However, the number of types of InPs and the probability with which a certain InP may belong to a certain type are known.

\section{Contract With Complete Information}

Consider a complete information scenario, where the MVNO knows precisely the type of the InPs so as the BSs. In the complete information scenario, as the types of InPs are related to the SNR of the UEs that they are connected with, the MVNO can obtain precise information about the type of each InP by the feedback of the UEs. However, such an assumption may not be practical as additional communications cost occurs and requires high real time communications. We use the maximum MVNO's utility achieved in this case as a benchmark to evaluate the performance of the proposed contracts under incomplete information in the following subsection.

As the MVNO knows each InP's type, the MVNO can centrally monitor and make sure that each InP accepts only the contract item designed for its type. The MVNO needs to ensure that the InPs have non-negative payoffs in the contract designed for each type so that they are willing to accept the contract. In other words, the contract needs to satisfy the following defined Individual Rationality (IR) constraint when the contract is designed.

Definition 2. Individual Rationality (IR): The contract that an InP selects guarantees that the corresponding payoff is nonnegative, i.e.,

$$
x_{i}^{k}\left(\theta_{i}^{k} R\left(p_{i}^{k}\right)-e_{o}-\Psi\left(p_{i}^{k}\right)\right) \geq 0 .
$$

For notation simplicity, in the following, we use $\pi_{i}^{k}$ to denote $\pi\left(x_{i}^{k}, \theta_{i}^{k}, p_{i}^{k}\right)$ and $e_{i}^{k}$ to denote $e\left(x_{i}^{k}, p_{i}^{k}\right)$. Further, we denote $v_{i}^{k}=v\left(x_{i}^{k}, p_{i}^{k}\right)=x_{i}^{k} R\left(p_{i}^{k}\right)$.

We assume that $N_{i}^{k} \geq 1$ is the number of type- $\theta_{i}^{k}$ InPs, which should be known to the MVNO. Then, in the complete information scenario, an optimal contract maximizing the InPs' payoff is given as follows:

$$
\text { P1: } \max _{\{\mathbf{x}, \mathbf{p}\}} \sum_{i \in \mathcal{I}} \sum_{k \in \mathcal{K}} N_{i}^{k} \varpi_{i}
$$

$$
\begin{aligned}
& \text { s.t. } \mathbf{C} 1: x_{i}^{k}=\{0,1\}, 0<\sum_{k \in \mathcal{K}} x_{i}^{k} \leq 1, \forall i \in \mathcal{I}, \\
& \mathbf{C} 2: 0 \leq \sum_{i \in \mathcal{I}} x_{i}^{k} p_{i}^{k} \leq p_{\text {max }}^{k}, \forall i \in \mathcal{I}, \forall k \in \mathcal{K}, \\
& \text { C3 }: \pi_{i}^{k}-e_{i}^{k} \geq 0, \forall k \in \mathcal{K} .
\end{aligned}
$$

In (19), $\mathbf{C} \mathbf{1}$ shows that one UE can be served by one InP. It can also ensure that one UE can be associated with at most one InP. C2 is the transmit power constraint, which means that the transmit power should be less than its maximum power $p_{\max }^{k}$. C3 represents the IR constraints. As can be found, due to the defined user association indicator, the problem P1 is a NP-hard non-convex mixed integer non-linear programming (MINLP) optimization problem, of which the optimal solution is computationally expensive. For the immediate NP-hardness proof of P1, given that the association indicator $x_{i}^{k}$ can be either 0 or 1 , any feasible solution to P1 is a subset of vertices. Therefore, the solution to $\mathbf{P 1}$ describes a vertex cover, for which finding a maximum is NP hard. Interestingly, the formulated problem P1 can be viewed as a transformed energy efficient user association and power allocation problem in a multiuser network. In related works, e.g., [18] and [21], recursive methods are applied to obtain the optimal solutions, while some works consider using optimization theoretic schemes to solve the problem, e.g., in [20] and [22]. In addition to the heuristic algorithms that can search for the optimal solution, to address such an MINLP problem, we first relax the binary variable $x_{i}^{k}$ to be a real positive value between 0 and 1 , i.e., $x_{i}^{k} \in[0,1]$. The relaxed $x_{i}^{k}$ can be interpreted as the resource sharing factor represents the ratio of time when $\mathrm{UE} i$ associates to the $\mathrm{BS} / \mathrm{InP} k$. Then, the corresponding problem can be transformed a convex optimization problem and addressed accordingly. The solution procedure can be found in e.g., [25], [26], [28] and others. In short, the convexity of P1 can be proved, and Karush-Kuhn-Tucker (KKT) conditions can be applied to find the optimal solution of $x_{i}^{k}$ and $p_{i}^{k}$.

Note that the optimal $x_{i}^{k}$ obtained by the proposed relaxation is actually the upper bound of the optimization problem P1, and we have to take the integer value due to the practical consideration that one UE can associate with one BS at a time [28]. After obtaining the contract item $\{\mathbf{x}, \mathbf{p}\}$, the MVNO assigns the contract and passes these items to the InPs. Once the contract is specified, each InP selects the contract item according to (16), i.e., the one that maximizes its payoff.

\section{CONTRACT WITH ASYMmETRIC INFORMATION}

In the case of asymmetric information, the types of InPs are not known to the MVNO. However, the number of InPs and the probability with which a certain InP may belong to a certain type are known [24], which is a very practical case when the MVNO is able to collect long-term feedback from the UEs. Therefore, we assume the MVNO has knowledge of the probability distribution that a certain BS/InP is type $-\theta_{i}^{k}$.

As discussed, we want to design a contract such that type $-\theta_{i}^{k}$ InP would prefer the contract over all the others. The InP of type- $\theta_{i}^{k}$ will select the contract item $\left(x_{i}^{k}, p_{i}^{k}\right)$ by maximizing its own utility. Thus, the contract is known to be 
as a self-revealing contract if and only if (iff) the following constraint is satisfied.

Definition 3. Incentive Compatible (IC): InPs must prefer the contract designed specifically for their own types than the others, i.e.,

$$
\begin{aligned}
& \pi\left(x_{i}^{k}, \theta_{i}^{k}, p_{i}^{k}\right)-e\left(x_{i}^{k}, p_{i}^{k}\right) \geq \pi\left(x_{i}^{m}, \theta_{i}^{m}, p_{i}^{m}\right)-e\left(x_{i}^{m}, p_{i}^{m}\right), \\
& \forall k, m \in \mathcal{K} .
\end{aligned}
$$

In a scenario with asymmetric information, the optimal contract should satisfy not only the IR conditions but also the IC constraint. That is, the InP should have enough incentive to select the contract designed for InP's types. Then, the optimal contract in a scenario with asymmetric information is formulated as

$$
\begin{gathered}
\text { P2: } \max _{\{\mathbf{x}, \mathbf{p}\}} \sum_{i \in \mathcal{I}} \sum_{k \in \mathcal{K}} \mathbb{E}\left\{N_{i}^{k} \varpi_{i}\right\} \\
\text { s.t. } \widetilde{\mathbf{C} 1}: x_{i}^{k}=\{0,1\}, 0<\sum_{k \in \mathcal{K}} x_{i}^{k} \leq 1, \forall i \in \mathcal{I}, \\
\widetilde{\mathbf{C} 2}: 0 \leq \sum_{i \in \mathcal{I}} x_{i}^{k} p_{i}^{k} \leq p_{\text {max }}^{k}, \forall i \in \mathcal{I}, \forall k \in \mathcal{K}, \\
\widetilde{\mathbf{C} 3}: \pi_{i}^{k}-e_{i}^{k} \geq 0, \forall k \in \mathcal{K}, \\
\widetilde{\mathbf{C} 4}: \pi_{i}^{k}-e_{i}^{k} \geq \pi_{i}^{m}-e_{i}^{m}, \forall k, m \in \mathcal{K} .
\end{gathered}
$$

In (22), $\widetilde{\mathbf{C} 1}$ is the association constraint that one UE can be associated with at most one InP. $\widetilde{\mathrm{C} 2}$ is the constraint of the transmit power. $\widetilde{\mathrm{C} 3}$ and $\widetilde{\mathrm{C} 4}$ represent the IR and IC constraints, respectively. The IR constraint can ensure the InP has a non-negative payoff by choosing the contract designed for it, and IC constraint can make sure that for type- $\theta_{i}^{k}$ InP, it can get the maximum payoff by selecting the contract designed for it. First, we provide the feasibility analysis of the contract.

\subsection{Feasibility of the Contract}

Next, we derive the conditions to ensure a feasible contract that satisfies the IR and IC constraints in the case with asymmetric information.

Lemma 1. : For any feasible contract $\mathbb{C}, v_{i}^{k}>v_{i}^{m}$ iff $\theta_{i}^{k}>\theta_{i}^{m}$, and $v_{i}^{k}=v_{i}^{m}$ iff $\theta_{i}^{k}=\theta_{i}^{m}$.

Proof. We prove this Lemma by using the IC constraint in (20) and by contradiction. From the IC constraint, we can see

$$
\begin{aligned}
& \theta_{i}^{k} v_{i}^{k}-e_{i}^{k}>\theta_{i}^{m} v_{i}^{m}-e_{i}^{m}, \\
& \theta_{i}^{m} v_{i}^{m}-e_{i}^{m}>\theta_{i}^{m} v_{i}^{k}-e_{i}^{k} .
\end{aligned}
$$

Then, we suppose there exists a contract with $\theta_{i}^{k}>\theta_{i}^{m}$ and $v_{i}^{k}<v_{i}^{m}$. Accordingly, we have

$$
\left(\theta_{i}^{k}-\theta_{i}^{m}\right)\left(v_{i}^{k}-v_{i}^{m}\right)<0
$$

and thus,

$$
\theta_{i}^{k} v_{i}^{k}+\theta_{i}^{m} v_{i}^{m}<\theta_{i}^{m} v_{i}^{k}+\theta_{i}^{k} v_{i}^{m} .
$$

However, combining (23) and (24), we have

$$
\theta_{i}^{k} v_{i}^{k}+\theta_{i}^{m} v_{i}^{m}>\theta_{i}^{m} v_{i}^{k}+\theta_{i}^{k} v_{i}^{m}
$$

which is contradicted with (26). Therefore, if $\theta_{i}^{k}>\theta_{i}^{m}, v_{i}^{k}>$ $v_{i}^{m}$, which proves Lemma 1.

From Lemma 1, we know that if $\theta_{i}^{k}>\theta_{i}^{m}$ holds, then $v_{i}^{k}>v_{i}^{m}$ must hold. Thus, an InP of a higher type should receive more reward than an InP of lower type. If two InPs receive the same utility, they must belong to the same type and vice versa. Given Definition 1, we can have $\left.v_{i}^{1}<\ldots<v_{i}^{k}<v_{i}^{K}\right)$. Correspondingly, we have the following definition.

Definition 4. Monotonicity: For any feasible contract $\mathbb{C}$, the utility of the InP follows,

$$
0 \leq v_{i}^{1}<\ldots<v_{i}^{k}<\ldots<v_{i}^{K} .
$$

Such a definition implies that the InPs of a higher type have a higher willingness to offer services and participate in the contract. With the property in monotonicity, we also have the following proposition.

Proposition 1. With a strictly increasing utility, the cost satisfies the following condition:

$$
0 \leq e_{i}^{1}<\ldots<e_{i}^{k}<\ldots<e_{i}^{K} .
$$

Proposition 1 shows that an incentive-compatible contract requires a higher contribution of an InP if it receives a high utility and vice versa.

Lemma 2. For any feasible contract $\mathbb{C}$, the payoff of the InP follows,

$$
0 \leq U_{1}<\ldots<U_{k}<\ldots<U_{K} .
$$

Proof. From the definition of Monotonicity and Proposition 1, we can see that InPs that ask for more utility must be able to provide larger transmit power (correspondingly throughput in (2)). If $\theta_{i}^{k}>\theta_{i}^{m}$, we have

$$
\theta_{i}^{k} v_{i}^{k}-e_{i}^{k}>\theta_{i}^{k} v_{i}^{m}-e_{i}^{m}>\theta_{i}^{m} v_{i}^{m}-e_{i}^{m} .
$$

Thus, we can have $U_{k}>U_{m}$, if $\theta_{i}^{k}>\theta_{i}^{m}$.

As we can see, the InPs of higher types receive a better payoff than the ones of lower types. From the IC constraint and the two lemmas above, we can easily deduce the following. If an InP of a high type selects the contract designed for the InP of a low type, even though a smaller transmit power and throughput are required, the less utility received will deteriorate its payoff. Moreover, if an InP of a low type selects a contract designed for an InP of a high type, the gain in terms of utility cannot compensate the cost in terms of transmit power consumption. The InPs can receive the maximum payoff iff they select the contract that designed according to their types. Thus, the self-reveal property of a contract can be ensured. Moreover, we can have the following properties.

Lemma 3. For any contract $\mathbb{C}$, only one contract item of the highest type $\theta_{i}^{K}$ can have positive user association, and all other items should have zero association, i.e.,

$$
\begin{cases}x_{i}^{k} \leq 1, & \text { if } k=K, \\ x_{i}^{k}=0, & \text { otherwise. }\end{cases}
$$


Proof. From $\widetilde{\mathbf{C} 1}$, we can see that for any UE $i$, there can be only one contract that $x_{i}^{k}=1$ and the others should equal to zero. Then, we prove that only the item of the highest type can have a positive value by contradiction. Suppose there exists a contract with item $x_{i}^{m}>0$ and $1<m<K$. Then, because of the constraints of $\widetilde{\mathbf{C}}$, all the other contract items $x_{i}^{k}=0, \forall k \neq m$, which means, $v_{i}^{K} \leq v_{i}^{m}$ and $m<K$. Such an observation contradicts Lemma 1 and monotonicity.

Lemma 3 indicates that for a feasible contract the UE will be associated with the InP of the highest type. Based on the properties described above, we could arrive to the sufficient and necessary conditions in Theorem $\mathbf{1}$ for feasibility.

Theorem 1. For any feasible contract $\mathbb{C}$, it is feasible iff the following conditions are satisfied.

$$
\begin{array}{ll}
\text { L1 : } & 0=v_{i}^{1}=\ldots=v_{i}^{k}=\ldots<v_{i}^{K}, \text { and } \\
& 0=e_{i}^{1}=\ldots=e_{i}^{k}=\ldots<e_{i}^{K}, \\
\text { L2 : } & \pi_{i}^{1} \geq e_{i}^{1} \geq 0, \\
\text { L3 : } & \theta_{i}^{k-1}\left(v_{i}^{k}-v_{i}^{k-1}\right)+e_{i}^{k-1} \leq e_{i}^{k} \\
& \leq \theta_{i}^{k}\left(v_{i}^{k}-v_{i}^{k-1}\right)+e_{i}^{k-1}
\end{array}
$$

\subsection{Optimal Contract Design}

In this case, the MVNO does not know the exact type of each InP, but knows the number of InPs and the probability with which a certain InP may belong to a certain type. Such a probability can be denoted as $\rho_{i}^{k}=\operatorname{Pr}\left\{\theta_{i}^{k}=\theta_{i j}^{n}\right\}, \forall n \in$ $\mathcal{N}_{j}, \forall j \in \mathcal{J}$. Obviously, $\sum_{k \in \mathcal{K}} \rho_{i}^{k}=1, \forall i \in \mathcal{I}$. Before addressing the formulated problem, we first present the following propositions.

Proposition 2. If the IR constraint of type- $\theta_{i}^{1} \operatorname{InP}$ is satisfied, the other IR constraints will automatically hold.

Proof. From Definition 1, we have $\theta_{i}^{1}<\cdots<\theta_{i}^{k}<\cdots \theta_{i}^{K}$. Then by using IC constraints, we have

$$
\begin{aligned}
& \pi\left(x_{i}^{k}, \theta_{i}^{k}, p_{i}^{k}\right)-e\left(x_{i}^{k}, p_{i}^{k}\right) \geq \pi\left(x_{i}^{1}, \theta_{i}^{k}, p_{i}^{1}\right)-e\left(x_{i}^{1}, p_{i}^{1}\right) \\
& \geq \pi\left(x_{i}^{1}, \theta_{i}^{1}, p_{i}^{1}\right)-e\left(x_{i}^{1}, p_{i}^{1}\right), \forall k, m \in \mathcal{K} .
\end{aligned}
$$

Thus, we can see that if the IR constraint of type- $\theta_{i}^{1} \operatorname{InP}$ is satisfied, the other IR constraints will hold as well.

Accordingly, we can simply replace $\widetilde{\mathbf{C} 3}$ and keep the first IR constraints and reduce the others. Next, we can reduce the IC constraints by proposing the following propositions.

Proposition 3. If the Local Downward Incentive Constraints (LDICs) are satisfied for all $\operatorname{InP}$ type $\theta_{i}^{k}$, i.e.,

$$
\begin{aligned}
& \pi\left(x_{i}^{k}, \theta_{i}^{k}, p_{i}^{k}\right)-e\left(x_{i}^{k}, p_{i}^{k}\right) \geq \\
& \pi\left(x_{i}^{k-1}, \theta_{i}^{k}, p_{i}^{k-1}\right)-e\left(x_{i}^{k-1}, p_{i}^{k-1}\right) .
\end{aligned}
$$

The IC constraint can hold for $m \geq k$, i.e.,

$$
\pi\left(x_{i}^{k}, \theta_{i}^{k}, p_{i}^{k}\right)-e\left(x_{i}^{k}, p_{i}^{k}\right) \geq \pi\left(x_{i}^{m}, \theta_{i}^{k}, p_{i}^{m}\right)-e\left(x_{i}^{m}, p_{i}^{m}\right) .
$$

Proof. Consider three types of InPs $\theta_{i}^{k-1}<\theta_{i}^{k}<\theta_{i}^{k+1}$, and we obtain

$$
\begin{aligned}
& \pi\left(x_{i}^{k+1}, \theta_{i}^{k+1}, p_{i}^{k+1}\right)-e\left(x_{i}^{k+1}, p_{i}^{k+1}\right) \geq \\
& \pi\left(x_{i}^{k}, \theta_{i}^{k+1}, p_{i}^{k}\right)-e\left(x_{i}^{k}, p_{i}^{k}\right),
\end{aligned}
$$

and

$$
\begin{aligned}
& \pi\left(x_{i}^{k}, \theta_{i}^{k}, p_{i}^{k}\right)-e\left(x_{i}^{k}, p_{i}^{k}\right) \geq \\
& \pi\left(x_{i}^{k-1}, \theta_{i}^{k}, p_{i}^{k-1}\right)-e\left(x_{i}^{k-1}, p_{i}^{k-1}\right) .
\end{aligned}
$$

In Lemma 1, we can see that $v_{i}^{k} \geq v_{i}^{m}$ whenever $\theta_{i}^{k} \geq$ $\theta_{i}^{m}$, then (38) becomes

$$
\begin{gathered}
\theta_{i}^{k+1}\left(v_{i}^{k}-v_{i}^{k-1}\right) \geq \theta_{i}^{k}\left(v_{i}^{k}-v_{i}^{k-1}\right) \geq e_{i}^{k}-e_{i}^{k-1}, \\
\theta_{i}^{k+1} v_{i}^{k+1}-e_{i}^{k+1} \geq \theta_{i}^{k+1} v_{i}^{k}-e_{i}^{k} \geq \theta_{i}^{k+1} v_{i}^{k-1}-e_{i}^{k-1} .
\end{gathered}
$$

(39) and (40) imply that for InP type $\theta_{i}^{k+1}$, besides $\left(x_{i}^{k}, p_{i}^{k}\right)$, the LDIC is satisfied for contract item $\left(x_{i}^{k-1}, p_{i}^{k-1}\right)$. By iterating, it can be deduced the LDIC holds for all contract items $\left(x_{i}^{m}, p_{i}^{m}\right)$ when $m \leq k$, indicating that the IC constraint is satisfied. In view of a random selection of $\theta_{i}^{k+1}$, we have completed the proof.

Proposition 4. : If the Local Upward Incentive Constraints (LUICs) are satisfied for all InP type $\theta_{i}^{k}$, i.e.,

$$
\begin{aligned}
& \pi\left(x_{i}^{k}, \theta_{i}^{k}, p_{i}^{k}\right)-e\left(x_{i}^{k}, p_{i}^{k}\right) \geq \\
& \pi\left(x_{i}^{k+1}, \theta_{i}^{k}, p_{i}^{k+1}\right)-e\left(x_{i}^{k+1}, p_{i}^{k+1}\right) .
\end{aligned}
$$

The IC contraint can hold for $m \geq k$, i.e.,

$$
\pi\left(x_{i}^{k}, \theta_{i}^{k}, p_{i}^{k}\right)-e\left(x_{i}^{k}, p_{i}^{k}\right) \geq \pi\left(x_{i}^{m}, \theta_{i}^{k}, p_{i}^{m}\right)-e\left(x_{i}^{m}, p_{i}^{m}\right) .
$$

Proof. The proof is similar to the proof of Proposition 3, so we omit it here.

With the previous analysis, we can have following proposition to release the IC constraints.

Proposition 5. : If the payoff of the MVNO is maximized, i.e., the optimal contract design is obtained, then the LDICs must satisfy the following condition, i.e.,

$$
\begin{aligned}
& \pi\left(x_{i}^{k}, \theta_{i}^{k}, p_{i}^{k}\right)-e\left(x_{i}^{k}, p_{i}^{k}\right)= \\
& \pi\left(x_{i}^{k-1}, \theta_{i}^{k}, p_{i}^{k-1}\right)-e\left(x_{i}^{k-1}, p_{i}^{k-1}\right) .
\end{aligned}
$$

Proof. Suppose the LDICs hold for any type of $\operatorname{InP} \theta_{i}^{k}$. The LDICs will still be satisfied if $e\left(x_{i}^{k}, p_{i}^{k}\right)$ and $e\left(x_{i}^{k-1}, p_{i}^{k-1}\right)$ are raised by the same positive amount. To maximize its payoff, the MVNO will try to raise the use of radio resources for as much as possible until the following equation holds, i.e., $\pi\left(x_{i}^{k}, \theta_{i}^{k}, p_{i}^{k}\right)-e\left(x_{i}^{k}, p_{i}^{k}\right)=\pi\left(x_{i}^{k-1}, \theta_{i}^{k}, p_{i}^{k-1}\right)-$ $e\left(x_{i}^{k-1}, p_{i}^{k-1}\right)$. Note that this process will not impact the other LDICs. Therefore, if the contract is at the optimum, the condition will hold.

In fact, combining the LDICs in Proposition 4 and the monotonicity condition, we can conclude that all the LUICs will hold. That is, $\pi\left(x_{i}^{k}, \theta_{i}^{k}, p_{i}^{k}\right)-$ $e\left(x_{i}^{k}, p_{i}^{k}\right)=\pi\left(x_{i}^{k-1}, \theta_{i}^{k}, p_{i}^{k-1}\right)-e\left(x_{i}^{k-1}, p_{i}^{k-1}\right)$ implies that $\pi\left(x_{i}^{k}, \theta_{i}^{k}, p_{i}^{k}\right)-e\left(x_{i}^{k}, p_{i}^{k}\right) \geq \pi\left(x_{i}^{k-1}, \theta_{i}^{k}, p_{i}^{k-1}\right)-$ $e\left(x_{i}^{k-1}, p_{i}^{k-1}\right)$. Therefore, the IC constraint can be replaced by the LDICs and the monotonicity condition.

Consequently, to find a feasible and optimal solution, P2 can be reformed as

$$
\text { P3: } \max _{\{\mathbf{x}, \mathbf{p}\}} \sum_{i \in \mathcal{I}} \sum_{k \in \mathcal{K}} \rho_{i}^{k} N \varpi_{i}
$$




$$
\begin{aligned}
& \text { s.t. } \widetilde{\mathbf{C} 1}: x_{i}^{k}=\{0,1\}, 0<\sum_{k \in \mathcal{K}} x_{i}^{k} \leq 1, \forall i \in \mathcal{I}, \\
& \widetilde{\mathbf{C} 2}: 0 \leq \sum_{i \in \mathcal{I}} x_{i}^{k} p_{i}^{k} \leq p_{\text {max }}^{k}, \forall i \in \mathcal{I}, \forall k \in \mathcal{K}, \\
& \widetilde{\mathbf{C} 3}: \pi_{i}^{1}-e_{i}^{1}=0, \forall k \in \mathcal{K}, \\
& \widetilde{\mathbf{C} 4}: \theta_{i}^{k} v_{i}^{k}-e_{i}^{k}=\theta_{i}^{k} v_{i}^{k-1}-e_{i}^{k-1}, \forall k \in \mathcal{K}, \\
& \widetilde{\mathbf{C} 5}: \sum_{k \in \mathcal{K}} \rho_{i}^{k}=1, \forall i \in \mathcal{I} . \\
& \widetilde{\mathbf{C} 6}: 0 \leq v_{i}^{1}<\ldots<v_{i}^{k}<\ldots<v_{i}^{K} .
\end{aligned}
$$

Similar to P1, P3 is also an NP-hard MINLP optimization problem. To solve this problem, we can formulate and solve the relaxed problem without the monotonicity condition. Then, we can follow the procedure for solving P1. We first relax the binary variable $x_{i}^{k}$ to be a real positive value between 0 and 1, i.e., $x_{i}^{k} \in[0,1]$. Then, the corresponding problem can be transformed into a convex optimization problem and addressed accordingly, i.e., KKT conditions can be applied to find the optimal solutions for $x_{i}^{k}$ and $p_{i}^{k}$.

Then we check whether the solution to this relaxed problem guarantees the monotonicity condition or not. The optimal contract resulting from this optimization problem will yield zero payoff for the lowest type of InPs. If $K=2$, there are only two types of InPs, the high type and the low type. By solving this optimization problem, the low type InPs will obtain a zero payoff contract, and only the high type UEs can receive a positive utility. In the general cases when $K>2$, all types of InPs will get a zero payoff except the highest type that will get a positive payoff, where a similar conclusion is also provided in [18] and [20].

\subsection{Algorithm Implementation}

By solving the proposed problems, we can provide the InPs with the optimal contracts that can stimulate and encourage the InPs to provide the resource. To implement the proposed approach in a practical WVN, the following steps can be considered. Once there are some UEs that have service requirements, the MVNO will offer the contracts to the InPs. After signing the contracts, the signed InPs will set up the connections with the UEs and start the transmission. The MVNO will stand by and wait for the feedback of the services. If the service or transmission is successful, the MVNO will pay the involved InP based on the signed contract. Otherwise, if the connection fails, the MVNO may need to find other InPs to serve the UEs, and the InPs with failure links will not receive payment. The proposed user association and resource allocation algorithm is summarized in Algorithm 1, which presents the practical implementation.

Note that in some cases, the optimal $x_{i}^{k}$ obtained by the proposed relaxation is actually the upper bound of the optimization problem P1 and P3, and we have to take the integer value due to practical consideration that one UE can associate with one BS at a time. In this work, we follow the algorithms in [26] and [27] during the optimization process to select the BS that can offer the best link quality for the UE, which is typical in the investigation of the resource allocation scheme. In addition, the iterative procedure can also

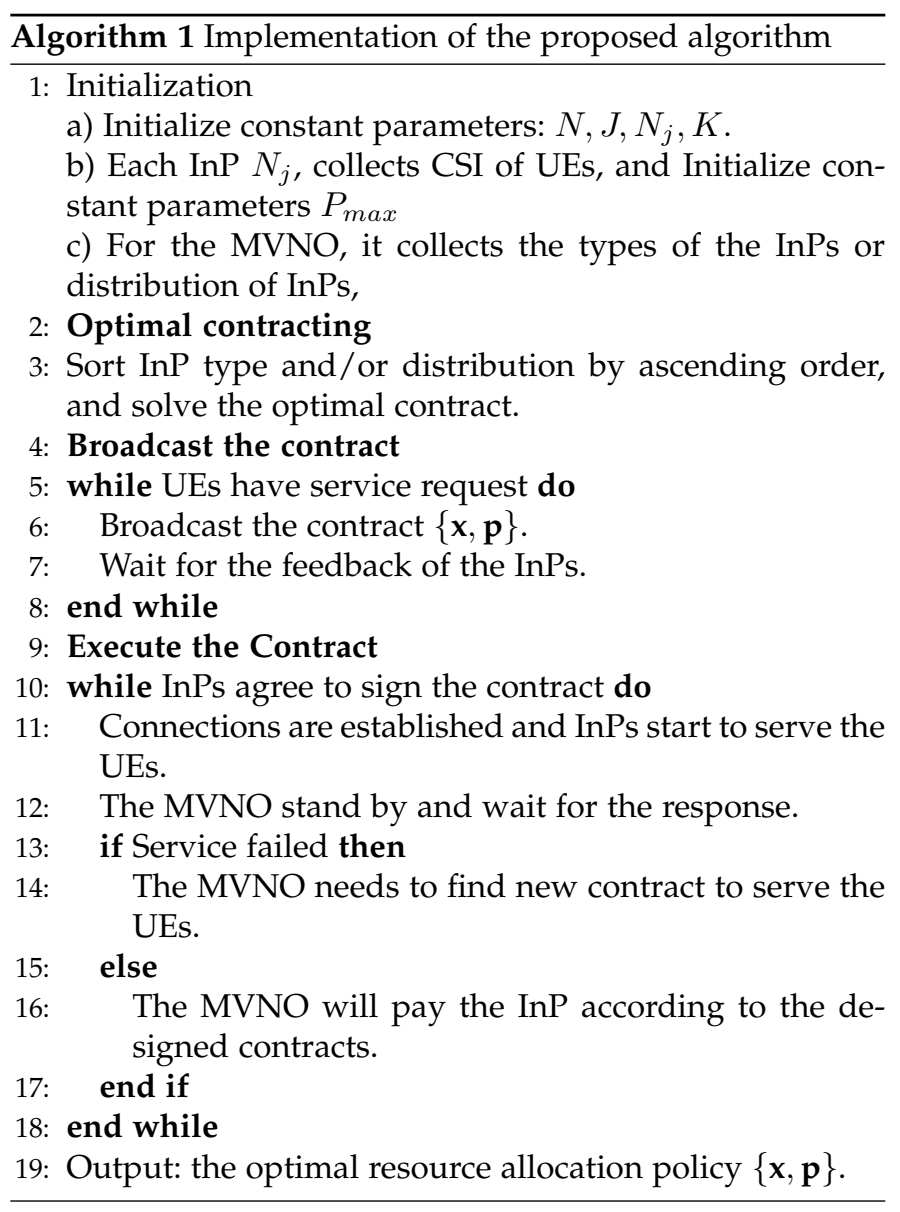

be applied to obtain the binary variables [28]. The process can start by assuming there is no association between the BS/InP and UE. In each iteration, $x_{i}^{k}$ and power allocation are addressed with a set of UE-BS associations that are not connected. The UE-BS connection with the largest value of $x_{i}^{k}$ will be made.

\section{Performance Evaluation}

In this section, the performance of the proposed scheme is evaluated and illustrated. For simplicity, we assume there are three InPs and they operate in a $100 \times 100 \mathrm{~m}^{2}$ area. There are 20 UEs in this area requesting the mobile service from the MVNO. It is considered that the UEs are randomly located. The maximum transmit power of the BS of the InP is $46 \mathrm{dBm}$ unless specified. To illustrate the effectiveness of the proposed scheme, we prefer to use simple and wellknown functions to model the cost and revenues. We define the quality-related cost $\Psi\left(p_{i}^{k}\right)$ in (8) as a linear cost related to the power consumption, i.e., $\Psi\left(p_{i}^{k}\right)=\tau p_{i}^{k}$, where $\tau$ is the cost parameter. Therefore, $\Psi\left(p_{i}^{k}\right)$ increments linearly with the cost of power consumption, and without being specified, we consider $\tau=1$.

In Fig. 2, with the consideration of complete information, i.e., the MVNO has the full knowledge of the types of InPs, we present the MVNO's utility with respect to the number of UEs and InP types. First, we can observe that as the number of types of InPs increases, the overall utility also gets larger. This is mainly due to the fact that the increase of the number of types of InPs essentially mean there are 


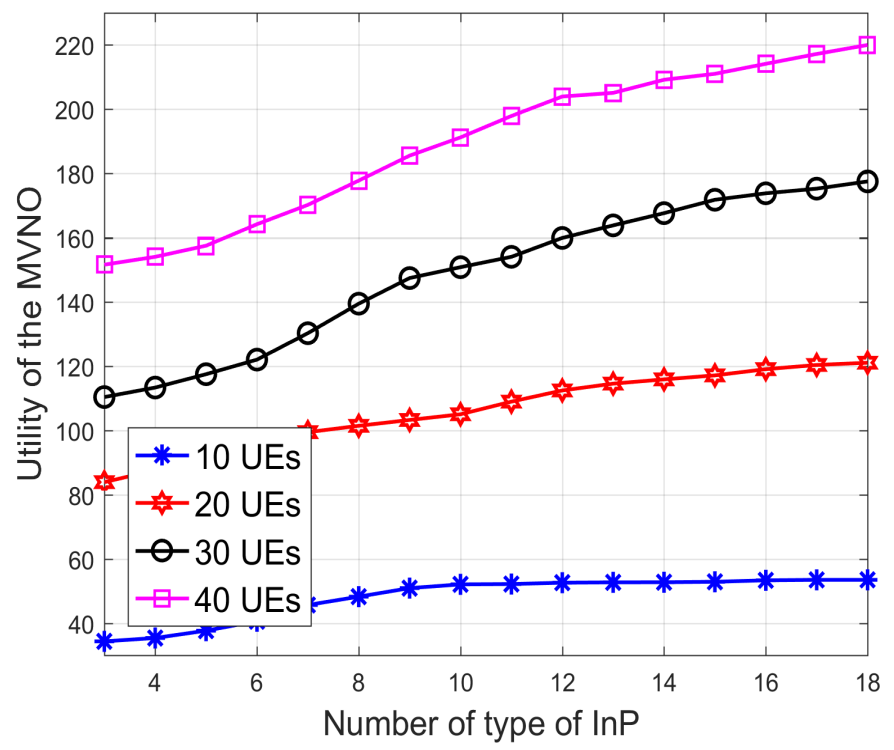

Fig. 2. MVNO's utility with respect to number of UEs and InP types, complete information case.

more and better options to provide better services to the UEs. Therefore, the utility, which is related to the data rate of the UEs, increases as well. However, when there are only 10 UEs in the system, the increment of the utility becomes slow when the number of types of InPs is above 10. This may due to the fact that in this area, there are enough number of types of InPs for the UEs. Simply increasing the types of InPs will not improve the service quality, as the BSs of the InPs may be close enough to the UEs. When there are 20 UEs, the utility can be improved as the number of types of InPs without any saturation. However, the speed of increment becomes slow. For the cases with 30 and 40 UEs, the utility always increases when there are more types of InPs, which means there is still room for the service provisioning of the MVNO.

In Fig. 3, we have compared the utility performance of the contract with complete information and the one with asymmetric information. We also show a linear pricing mechanism here [20]. The linear pricing contract is under the information asymmetry. In this mechanism, the price offered by the MVNO is linear to the transmit power. It can be found in this figure that the MVNO achieves the highest utility when the types of the InPs are known. For the case with asymmetric information, the exact value of the type of the InP is unavailable to the BS. Thus, the MVNO can only achieve a near optimal utility at best only under information asymmetry, which is upper bounded by the complete information case. Meanwhile, the proposed solution for the contract with information asymmetry yields a better utility that the linear pricing case. As in the linear pricing case, the choices of the InPs are not restricted, and less information is retrieved. Therefore, it prevents the MVNO from obtaining more utility.

Then we evaluate the impact of the cost model on the system performance. There are 20 UEs and 10 types of InPs. Fig. 4 presents the MVNO's payoff and optimal contract with various $\tau$. We show the performance of the cases

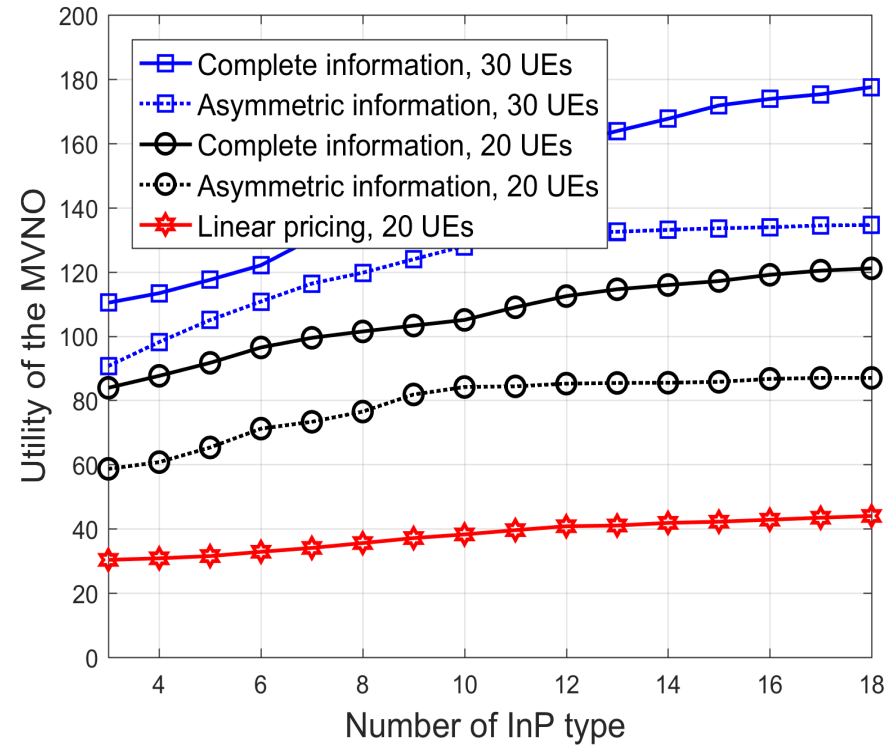

Fig. 3. MVNO's utility with respect to InP types, complete information case vs. asymmetric information, 10 and 30 UEs.

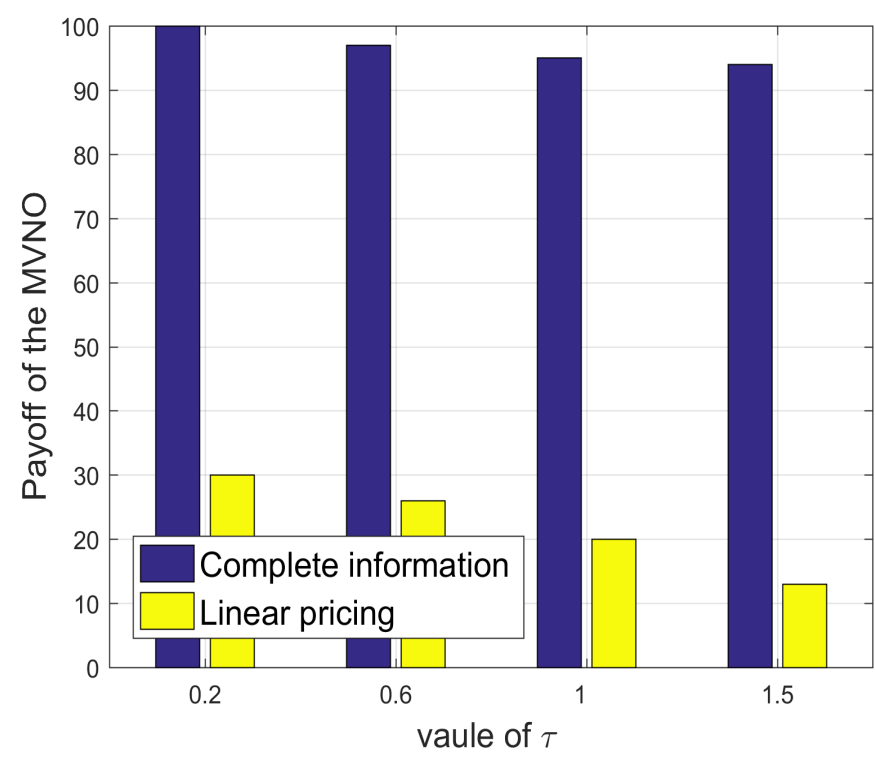

Fig. 4. MVNO's payoff with respect to value of $\tau$, complete information case vs. asymmetric information, 10 types of InPs and 20 UEs.

with complete information and linear pricing. Similar to the observation in Fig. 3, for the case with complete information we observe that InPs' payoff is larger than that in the case with linear pricing. When $\tau$ is small, the transmit power has less impact on the cost, and the InPs may prefer to offer a higher transmit power to get more utility in the optimal contract design. Therefore, the gap is not as big. When $\tau$ increases, this impact becomes larger, and InPs' transmit power may become smaller, which reflects on the service quality at the UE. In the proposed scheme, as the transmit power is the optimization variable, the overall changes on the utility of the InP (cost of the MVNO) will not be significant. However, for the case of linear pricing, 


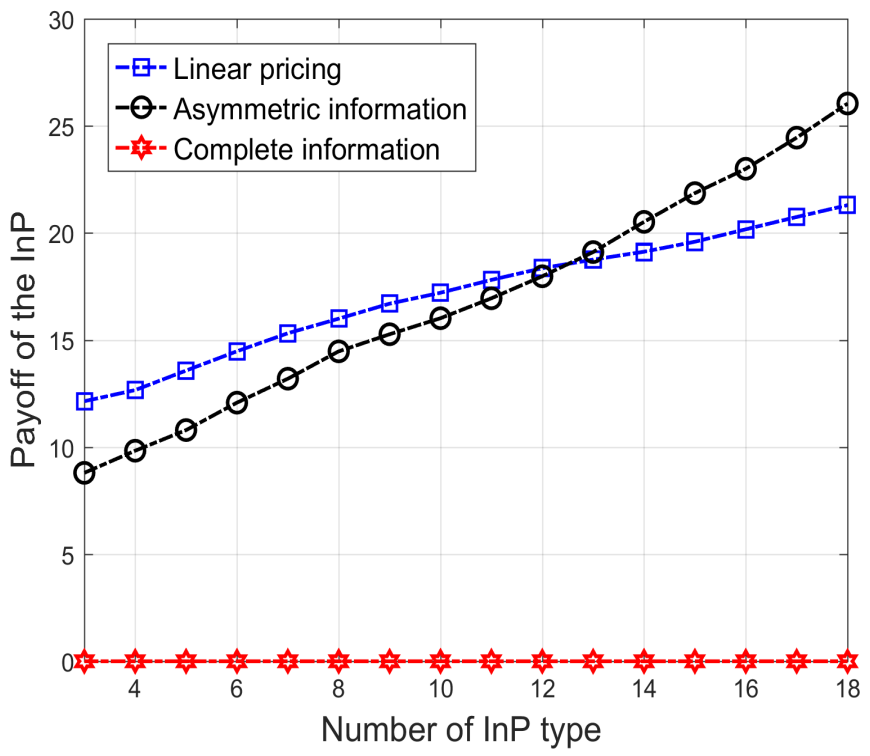

Fig. 5. InP's payoff with respect to number of InP types.

the effect is more serious, and thus, the gap becomes larger.

In Fig. 5, the payoff of the InP is presented. The result proves the monotonicity of the contract that the higher the type of InP, the larger the payoff it can receive under information asymmetry. All types of InPs enjoy a positive payoff. However, under complete information, the payoff remains 0 for all types of InPs, which is because when the MVNO is aware of the InP's type, it will adjust the contract to maximize its own payoff while leaving InPs with 0 payoff. Overall, it can be found that linear pricing brings the highest payoff, when the number of types of InPs is low. When the number is high, some of the higher type InPs may obtain higher payoffs from the optimal contract under information asymmetry than linear pricing.

In Fig. 6, we demonstrate the effect of $\tau$ on the power allocation at the algorithm level. On the x-axis of Fig. 6, we vary the maximum transmit power of the BS, e.g., $p_{\text {max }}^{k}$, and plot the corresponding average power consumption over all the BSs. As we can see, when $p_{\max }^{k}$ is small, the transmit power may not be able to provide the optimal value of P3. Therefore, the maximum transmit power is used. This phenomenon can be observed for all the values of $\tau$. As the maximum transmit power grows, optimal power allocation can be provided by the proposed scheme. It can be seen that a higher value of $\tau$ induces a lower optimal transmit power. However, when the value of $\tau$ is low, e.g., $\tau=0.2$, the transmit power is the highest, which also evidences the observation in Fig. 4.

\section{Conclusions and Future Research}

In this paper, a novel incentive mechanism is proposed to study how to manage the radio resources in the wireless virtualized networks. We consider that the InPs own the physical networks. The MVNO has the information of the users and need to lease the physical radio resources to provide services to users. In particular, we utilize the contract theoretic approach to model the resource trading

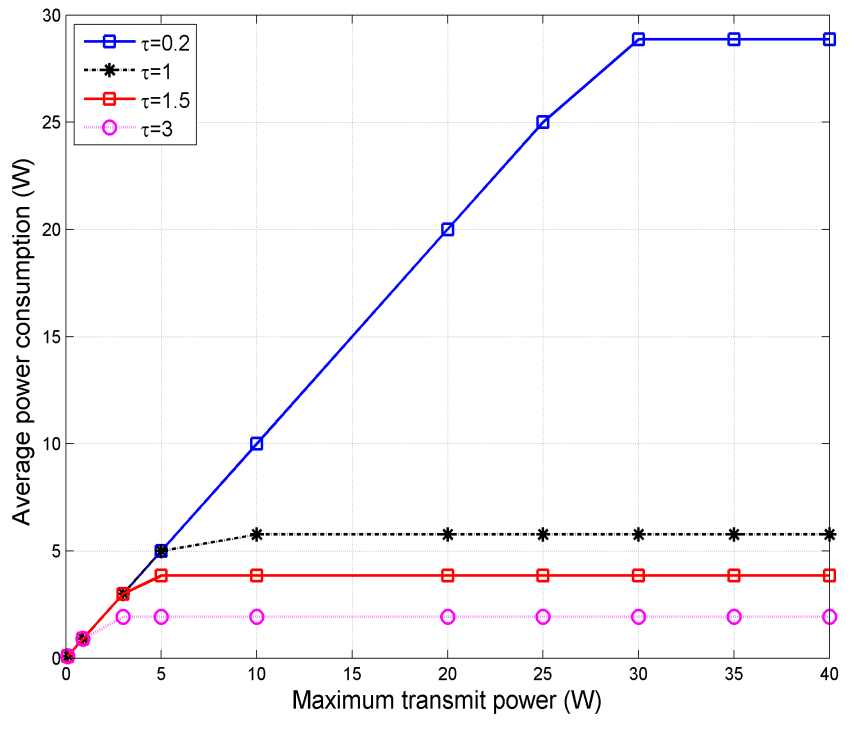

Fig. 6. Power allocation with respect to the value of $\tau$ and maximum transmit power, asymmetric information, 30 UEs.

and allocation process between an MVNO and multiple InPs. Two scenarios are considered according to whether the types of the InPs are globally known. Subsequently, the corresponding optimal contracts are derived to maximize the payoff of the MVNO while maintaining the requirements by the UEs in the trading process. To evaluate the proposed scheme, extensive simulation studies are conducted. It can be observed that the proposed contract theoretic approach can effectively stimulate InPs' participation, allocate the radio resources, and outperform other schemes.

In this work, we focus on a system with one MVNO and multiple InPs. As a future research direction, we will concentrate on the scenario containing multiple MVNOs and InPs. Accordingly, the interaction and competition among different MVNOs and the interactions among multiple MVNOs and InPs will be investigated utilizing novel network economic schemes.

\section{REFERENCES}

[1] F. Granelli, et al., "Software defined and virtualized wireless access in future wireless networks: scenarios and standards," IEEE Communications Magazine vol. 53, no. 6, pp. 26-34, June 2015.

[2] S. Abdelwahab, B. Hamdaoui, M. Guizani and T. Znati, "Network function virtualization in 5G," IEEE Communications Magazine, vol. 54, no. 4, pp. 84-91, April 2016.

[3] C. Liang and F. R. Yu, "Wireless network virtualization for next generation mobile cellular networks," IEEE Comm. Mag., vol. 22, no. 1, pp. 61-69, March 2015.

[4] Z. Feng, C. Qiu, Z. Feng, Z. Wei, W. Li and P. Zhang, "An effective approach to 5G: Wireless network virtualization," IEEE Communications Magazine, vol. 53, no. 12, pp. 53-59, Dec. 2015.

[5] H. K. Nguyen, Y. Zhang, Z. Chang, and Z. Han, "Parallel and distributed resource allocation with minimum traffic disruption for network virtualization," IEEE Trans. on Commun., vol. 65, no. 3, pp. 1162-1175, Mar. 2017.

[6] Z. Chang, Z. Han and T. Ristaniemi, "Energy efficient optimization for wireless virtualized small cell networks with large scale multiple antenna,"IEEE Trans. on Commun., vol. 65, no. 4, pp. 1696-1707, April 2017. 
[7] S. Parsaeefard, R. Dawadi, M. Derakhshani and T. Le-Ngoc, "Joint user-association and resource-allocation in virtualized wireless networks," IEEE Access, vol. 4, pp. 2738-2750, 2016.

[8] C. Liang, F. R. Yu, H. Yao and Z. Han, "Virtual resource allocation in information-centric wireless networks with virtualization," IEEE Trans. Veh. Technol., vol. 65, no. 12, pp. 9902-9914, Dec. 2016.

[9] K. Zhu, Z. Cheng, B. Chen and R. Wang, "Wireless virtualization as a hierarchical combinatorial auction: an illustrative example," in proc. of 2017 IEEE Wireless Communications and Networking Conference (WCNC), San Francisco, CA, April 2017.

[10] F. E. Zaheer, J. Xiao and R. Boutaba, "Multi-provider service negotiation and contracting in network virtualization," in proc. of 2010 IEEE Network Operations and Management Symposium (NOMS 2010), Osaka, Japan, 2010, pp. 471-478.

[11] D. Zhang, Z. Chang, T. Hämäläinen and F. R. Yu, "Double auction based multi-flow transmission in software-defined and virtualized wireless networks," IEEE Transactions on Wireless Communications, vol. 16, no. 12, pp. 8390-8404, Dec. 2017.

[12] D. H. N. Nguyen, Y. Zhang and Z. Han, "A Contract-theoretic approach to spectrum resource allocation in wireless virtualization," in proc. of 2016 IEEE Global Communications Conference (GLOBECOM), Washington, DC, 2016, pp. 1-6.

[13] G. Liu, F. R. Yu, H. Ji and V. C. M. Leung, "Virtual resource management in green cellular networks with shared full-duplex relaying and wireless virtualization: A game-Based approach," IEEE Transactions on Vehicular Technology, vol. 65, no. 9, pp. 7529-7542, Sept. 2016.

[14] D. B. Rawat, "Game theoretic approach for wireless virtualization with coverage and QoS constraints," 2017 IEEE Conference on Computer Communications Workshops (INFOCOM WKSHPS), Atlanta, GA, 2017, pp. 601-606.

[15] S. M. A. Kazmi, N. H. Tran, T. M. Ho and C. S. Hong, "Hierarchical matching game for service selection and resource purchasing in wireless network virtualization," IEEE Communications Letters, vol. 22, no. 1, pp. 121-124, Jan. 2018.

[16] T. D. Tran and L. B. Le, "Stackelberg game approach for wireless virtualization design in wireless networks," 2017 IEEE International Conference on Communications (ICC), Paris, France, 2017.

[17] P. Bolton, and M. Dewatripont, Contact theory, Cambridge, MA: The MIT Press, 2014.

[18] L. Gao, X. Wang, Y. Xu and Q. Zhang, "Spectrum trading in cognitive radio networks: a contract-theoretic modeling approach," IEEE Journal on Sel. Areas in Commun, vol. 29, no. 4, pp. 843-855, April 2011

[19] S. P. Sheng and M. Liu, "Profit incentive in trading nonexclusive access on a secondary spectrum market through contract design," IEEE/ACM Transactions on Networking, vol. 22, no. 4, pp. 1190-1203, Aug. 2014.

[20] Y. Zhang, L. Song, W. Saad, Z. Dawy and Z. Han, "Contractbased incentive mechanisms for device-to-device communications in cellular networks," IEEE Journal on Sel. Areas in Commun, vol. 33, no. 10, pp. 2144-2155, Oct. 2015.

[21] Y. Li, J. Zhang, X. Gan, L. Fu, H. Yu and X. Wang, "A contractbased incentive mechanism for delayed traffic offloading in cellular networks," IEEE Trans. on Wireless Commun., vol. 15, no. 8, pp. 53145327, Aug. 2016.

[22] T. Liu, J. Li, F. Shu and Z. Han, "Resource trading for a small-cell caching system: a contract-theory based approach," in proc. of 2017 IEEE Wireless Communications and Networking Conference (WCNC), San Francisco, CA, 2017, pp. 1-6.

[23] A. Asheralieva, and Y. Miyanaga, "Optimal contract design for joint user association and inter-cell interference mitigation in heterogeneous LTE-A networks with asymmetric information," IEEE Trans. Veh. Technol., vol. 66, no. 6, pp. 5284-5300, June 2017.

[24] C. J. Corbett, D. Zhou, and C. S. Tang, "Designing supply contracts: contract type and information asymmetry," Manage. Sci., vol. 50, no. 4, pp. 550-559, Apr. 2004

[25] Z. Chang, J. Gong, Y. Li, Z. Zhou, T. Ristaniemi, G. Shi, Z. Han and Z. Niu, "Energy efficient resource allocation for wireless power transfer enabled collaborative mobile clouds," IEEE Journal on Sel. Areas in Commun., vol. 34, no. 12, pp. 3438-3450, Dec. 2016.

[26] M. Wang, H. Gao and T. Lv, "Energy-efficient user association and power control in the heterogeneous network," IEEE Access, vol. 5, pp. 5059-5068, 2017.

[27] Z. Chang, T. Ristaniemi and Z. Niu, "Radio resource allocation for collaborative OFDMA relay networks with imperfect channel state information," IEEE Transactions on Wireless Communications, vol. 13, no. 5, pp. 2824-2835, May 2014.

[28] P. Luong, F. Gagnon, C. Despins, and Le-Nam Tran, "Optimal joint remote radio head selection and beamforming design for limited fronthaul c-ran," IEEE Trans. Signal Process., vol. 65, no. 21, pp. 56055620, Nov. 2017.

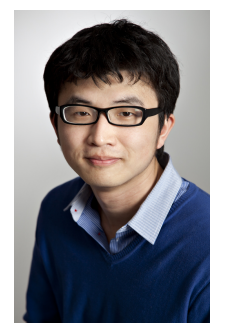

Zheng Changg (S'10-M'13-SM'17) received the B.Eng. degree from Jilin University, Changchun, China in 2007, M.Sc. (Tech.) degree from Helsinki University of Technology (Now Aalto University), Espoo, Finland in 2009 and Ph.D degree from the University of Jyväskylä, Jyväskylä, Finland in 2013. Since 2008, he has held various research positions at Helsinki University of Technology, University of Jyväskylä and Magister Solutions Ltd in Finland. During June to August in 2013, he was a visiting researcher at Tsinghua University, China and during April to May in 2015, he was a visiting researcher at University of Houston, TX. He has been awarded by the Ulla Tuominen Foundation, the Nokia Foundation and the Riitta and Jorma J. Takanen Foundation for his research excellence.

He serves as editor of IEEE Access, Springer Wireless Networks and IEEE MMTC Communications Frontier, and guest editor of IEEE Wireless Communications, IEEE Communications Magazine, IEEE Internet of Things Journal, EURASIP Journal on Wireless Communications and Networking, Physical Communications and Wireless Communications and Mobile Computing. He also served as TPC member for many IEEE major conferences, such as INFOCOM, ICC and Globecom. He has received Best Paper awards from IEEE Technical Committee on Green Communications \& Computing (TCGCC) and 23rd Asia-Pacific Conference on Communications (APCC) in 2017. He was also named the exemplary reviewer of IEEE Wireless Communications Letter in 2017. Currently he is working as Assistant Professor at University of Jyväskylä and his research interests include loT, cloud/edge computing, security and privacy, vehicular networks, and green communications.

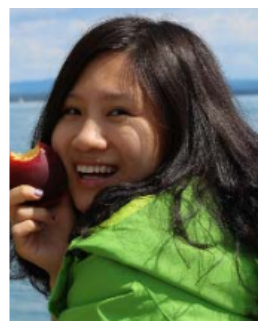

Di Zhang ) received the B.Eng. degree in automation in 2011 and the M.Eng. degree in system engineering from Northeastern University, Shenyang, China, in 2013. She is currently pursuing the Ph.D. degree in wireless communications with the University of Jyväskylä, Finland Her research work is funded by Chinese Scholarship Council, HPY Foundation, and Riitta and Jorma J. Takanen Foundation. Her research interests include game theoretic approaches, radio resource management, wireless virtualization, and software-defined networking

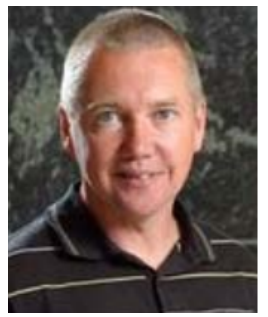

Timo Hämäläinen received the Ph.D. degree in telecommunication from the University of Jyväskylä, Finland, in 2002. He joined the University of Jyväskylä in 1997, where he is currently a Professor. He has over 25 years' research and teaching experience of computer networks. He has leaded tens of external funded network management related projects. He has launched and leads master programs with the University of Jyväskylä (SW \& Comm. Eng.), and teaches network management related courses. He has over 180 internationally peer reviewed publications and he has supervised almost $30 \mathrm{Ph} . \mathrm{D}$. theses. His research interests include network resource management, loT, and networking security. 


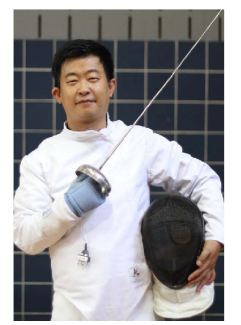

Zhu Han (S'01-M'04-SM'09-F'14) received the B.S. degree in electronic engineering from Tsinghua University, in 1997, and the M.S. and Ph.D. degrees in electrical and computer engineering from the University of Maryland, College Park, in 1999 and 2003, respectively.

From 2000 to 2002, he was an R\&D Engineer of JDSU, Germantown, Maryland. From 2003 to 2006, he was a Research Associate at the University of Maryland. From 2006 to 2008, he was an assistant professor at Boise State University, Idaho. Currently, he is a Professor in the Electrical and Computer Engineering Department as well as in the Computer Science Department at the University of Houston, Texas. His research interests include wireless resource allocation and management, wireless communications and networking, game theory, big data analysis, security, and smart grid. Dr. Han received an NSF Career Award in 2010, the Fred W. Ellersick Prize of the IEEE Communication Society in 2011, the EURASIP Best Paper Award for the Journal on Advances in Signal Processing in 2015, IEEE Leonard G. Abraham Prize in the field of Communications Systems (best paper award in IEEE JSAC) in 2016, and several best paper awards in IEEE conferences. Currently, Dr. Han is an IEEE Communications Society Distinguished Lecturer.

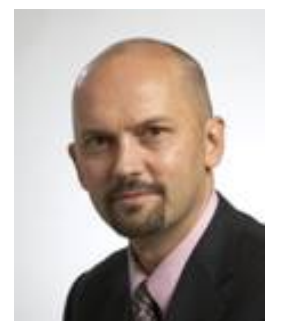

Tapani Ristaniemi received his M.Sc. in 1995 (Mathematics), Ph.Lic. in 1997 (Applied Mathematics) and Ph.D. in 2000 (Wireless Communications), all from the University of Jyväskylä, Jyväskylä, Finland. In 2001 he was appointed as Professor in the Department of Mathematical Information Technology, University of Jyväskylä. In 2004 he moved to the Department of Communications Engineering, Tampere University of Technology, Tampere, Finland, where he was appointed as Professor in Wireless Communications. In 2006 he moved back to University of Jyväskylä to take up his appointment as Professor in Computer Science. He is an Adjunct Professor of Tampere University of Technology. In 2013 he was a Visiting Professor in the School of Electrical and Electronic Engineering, Nanyang Technological University, Singapore.

He has authored or co-authored over 150 publications in journals, conference proceedings and invited sessions. He served as a Guest Editor of IEEE Wireless Communications in 2011 and currently he is an Editorial Board Member of Wireless Networks and International Journal of Communication Systems. His research interests are in the areas of brain and communication signal processing and wireless communication systems research. 